\title{
Cosmological Evolution of a Tachyon-Quintom Model of Dark Energy
}

\author{
Shang-Gang Shi ${ }^{1}$, Yun-Song Piao ${ }^{1}$ and Cong-Feng Qiao ${ }^{1,2}$ \\ ${ }^{1}$ College of Physical Sciences, Graduate University of CAS, YuQuan Road 19A, Beijing 100049, China \\ ${ }^{2}$ Theoretical Physics Center for Science Facilities (TPCSF), CAS., Beijing 100049, China
}

\begin{abstract}
In this work we study the cosmological evolution of a dark energy model with two scalar fields, i.e. the tachyon and the phantom tachyon. This model enables the equation of state $w$ to change from $w>-1$ to $w<-1$ in the evolution of the universe. The phase-space analysis for such a system with inverse square potentials shows that there exists a unique stable critical point, which has power-law solutions. In this paper, we also study another form of tachyon-quintom model with two fields, which voluntarily involves the interactions between both fields.
\end{abstract}




\section{INTRODUCTION}

Recent observational data [1, 2, 3] strongly indicate that the Universe is spatially flat and accelerating at the present time. Within the framework of general relativity, cosmic acceleration can be sourced by an energy-momentum tensor which has a large negative pressure called dark energy (See Ref. [4] for a recent review). The simplest candidate for dark energy seems to be a small positive cosmological constant, but it suffers from difficulties associated with the fine tuning and coincidence problem. This problem can be alleviated in models of dynamically evolving dark energy called quintessence [5], which have tracker like properties where the energy density in the fields track those of the background energy density before dominating today. The phantom, whose kinetic energy term has the reverse sign, has been also proposed as a candidate of dynamical dark energy [6]. There has been the enormous variety of DE models suggested in the literature, see Ref. [7] for reviews.

The analysis of the properties of dark energy from recent observations mildly favor models with $\omega$ crossing -1 in the near past [8, 9]. But, neither quintessence nor phantom can fulfill this transition. The quintom scenario of dark energy is designed to understand the nature of dark energy with $\omega$ across -1 . The first model of quintom scenario of dark energy is given in Ref. [9] with two scalar fields, where one is quintessence and the other is phantom. This model has been studied in detail later on [10, 11, 12, 13, 14, 15], and recently a new type of quintom model inspired by the string theory has also been proposed, which only have a single scalar field [16].

The role of the rolling tachyon [17 in string theory has been widely studied in cosmology, see Refs.[18, 19], and especially Refs. [20, 21, 22, 23] for dark energy. Some sort of tachyon condensate may described by effective field theory with a Lagrangian density $\mathcal{L}=-V(\phi) \sqrt{1+g^{\mu \nu} \partial_{\mu} \phi \partial_{\nu} \phi}$. It can act as a source of dark matter or inflation field. Meanwhile the tachyon can also act as a source of dark energy depending upon the form of the tachyon potential. However, as compared to canonical quintessence, tachyon models require more fine-tuning to agree with observations. In Ref. [24], the authors consider the BornInfeld type Lagrangian with negative kinetic energy term. The Lagrangian density they choose is $\mathcal{L}=-V(\varphi) \sqrt{1-g^{\mu \nu} \partial_{\mu} \varphi \partial_{\nu} \varphi}$. It is clear that for the spatially homogeneous scalar field, the equation of state $\omega=-1-\dot{\varphi}^{2}$ will be less than -1 unless the kinetic energy term $\dot{\varphi}^{2}=0$. This field is called phantom tachyon, see for example [25].

In principle, we can consider the multi-fields model including multiple tachyon and multiple phantom tachyon. However, without loss of generality, we only consider the case of one tachyon and one phantom tachyon, since it is the simplest one to realize that the equation of state $w$ cross -1 during the evolution of the universe and it shows most of the central ideas of such model. Because of the quintom-like behavior it shows, we call it tachyon-quintom for the convenience. This paper is organized as follows: in section II we study in detail the tachyon-quintom model with inverse square potentials. The numerical analysis shows this model is not sensitive to the initial kinetic energy density of tachyon and phantom tachyon, and we give the reason in detail. Then the phase-space analysis of the spatially flat FRW models shows that there exist a unique stable critical point, and we compare it 
with tachyon model; in section III we present another two-field model which include the interaction between two fields; the section IV is summary.

\section{THE TACHYON-QUINTOM MODEL}

\section{A. The tachyon-quintom model}

We assume a four-dimensional, spatially-flat Friedmann-Robertson-Walker Universe filled by a homogeneous tachyon $\phi$ with potential $V(\phi)$, a homogeneous phantom tachyon $\varphi$ with potential $V(\varphi)$ and a fluid with barotropic equation of state $p_{\gamma}=(\gamma-1) \rho_{\gamma}, 0<\gamma \leq 2$, such as radiation $(\gamma=4 / 3)$ or dust matter $(\gamma=1)$. In this section, we turn our attention to the possibility of the tachyon and phantom tachyon as a source of the dark energy.

The action for such a system is given by

$$
S=\int d^{4} x \sqrt{-g}\left(\frac{M_{p}^{2} \mathcal{R}}{2}+\mathcal{L}_{\phi}+\mathcal{L}_{\varphi}+\mathcal{L}_{m}\right)
$$

where $M_{p}$ is the reduced Planck mass, $\mathcal{R}$ is the scalar curvature, $\mathcal{L}_{m}$ represents the Lagrangian density of matter fields and

$$
\begin{aligned}
\mathcal{L}_{\phi} & =-V(\phi) \sqrt{1+g^{\mu \nu} \partial_{\mu} \phi \partial_{\nu} \phi} \\
\mathcal{L}_{\varphi} & =-V(\varphi) \sqrt{1-g^{\mu \nu} \partial_{\mu} \varphi \partial_{\nu} \varphi} .
\end{aligned}
$$

We now restrict to spatially homogeneous time dependent solutions for which $\partial_{i} \phi=\partial_{i} \varphi=$ 0 . Thus the energy densities and the pressure of the field $\phi$ and $\varphi$ are given, respectively, by

$$
\begin{aligned}
& \rho_{\phi}=\frac{V(\phi)}{\sqrt{1-\dot{\phi}^{2}}}, \quad p_{\phi}=-V(\phi) \sqrt{1-\dot{\phi}^{2}}, \\
& \rho_{\varphi}=\frac{V(\varphi)}{\sqrt{1+\dot{\varphi}^{2}}}, \quad p_{\varphi}=-V(\varphi) \sqrt{1+\dot{\varphi}^{2}}
\end{aligned}
$$

Here a dot is derivation with respect to synchronous time. The background equations of motion are

$$
\begin{gathered}
\frac{\ddot{\phi}}{1-\dot{\phi}^{2}}+3 H \dot{\phi}+\frac{1}{V(\phi)} \frac{d V(\phi)}{d \phi}=0 \\
\frac{\ddot{\varphi}}{1+\dot{\varphi}^{2}}+3 H \dot{\varphi}-\frac{1}{V(\varphi)} \frac{d V(\varphi)}{d \varphi}=0 \\
\dot{\rho}_{\gamma}=-3 \gamma H \rho_{\gamma} \\
\dot{H}=-\frac{1}{2 M_{p}^{2}}\left(\rho_{\phi}+p_{\phi}+\rho_{\varphi}+p_{\varphi}+\rho_{\gamma}+p_{\gamma}\right) \\
=-\frac{1}{2 M_{p}^{2}}\left(\frac{\dot{\phi}^{2} V(\phi)}{\sqrt{1-\dot{\phi}^{2}}}-\frac{\dot{\varphi}^{2} V(\varphi)}{\sqrt{1+\dot{\varphi}^{2}}}+\gamma \rho_{\gamma}\right)
\end{gathered}
$$


together with a constraint equation for the Hubble parameter:

$$
H^{2}=\frac{1}{3 M_{p}^{2}}\left(\frac{V(\phi)}{\sqrt{1-\dot{\phi}^{2}}}+\frac{V(\varphi)}{\sqrt{1+\dot{\varphi}^{2}}}+\rho_{\gamma}\right)
$$

The potentials we considered are inverse square potentials:

$$
V(\phi)=M_{\phi}^{2} \phi^{-2}, \quad V(\varphi)=M_{\varphi}^{2} \varphi^{-2}
$$

Those potentials allow constructing a autonomous system [26, 27] using the evolution equations, and give power-law solutions. The cosmological dynamics of the tachyon field with inverse square potential was studied in Ref. [20, 22, 23]. Interestingly, the inverse square potential plays the same role for tachyon fields as the exponential potential does for standard scalar fields.

We define the following dimensionless quantities:

$$
x_{\phi} \equiv \dot{\phi}, \quad y_{\phi} \equiv \frac{V(\phi)}{3 H^{2} M_{p}^{2}}, \quad x_{\varphi} \equiv \dot{\varphi}, \quad y_{\varphi} \equiv \frac{V(\varphi)}{3 H^{2} M_{p}^{2}}, \quad z \equiv \frac{\rho_{\gamma}}{3 H^{2} M_{p}^{2}}
$$

Now the Eqs. (10) and (91) can be rewrite as follow:

$$
\begin{aligned}
1 & =\frac{y_{\phi}}{\sqrt{1-x_{\phi}^{2}}}+\frac{y_{\varphi}}{\sqrt{1+x_{\varphi}^{2}}}+z \equiv \Omega_{D E}+z \\
\frac{H^{\prime}}{H} & =-\frac{3}{2}\left(-\frac{y_{\phi}\left(\gamma-x_{\phi}^{2}\right)}{\sqrt{1-x_{\phi}^{2}}}-\frac{y_{\varphi}\left(\gamma+x_{\varphi}^{2}\right)}{\sqrt{1+x_{\varphi}^{2}}}+\gamma\right)
\end{aligned}
$$

where $\Omega_{D E}$ measure the dark energy density as a fraction of the critical density, a prime denotes a derivative with respect to the logarithm of the scale factor, $N=\ln a$.

Then the evolution Eqs. (6) and (7) can be written to an autonomous system:

$$
\begin{gathered}
x_{\phi}^{\prime}=-3\left(x_{\phi}-\sqrt{\beta_{\phi} y_{\phi}}\right)\left(1-x_{\phi}^{2}\right) \\
y_{\phi}^{\prime}=3 y_{\phi}\left(-\frac{y_{\phi}\left(\gamma-x_{\phi}^{2}\right)}{\sqrt{1-x_{\phi}^{2}}}-\frac{y_{\varphi}\left(\gamma+x_{\varphi}^{2}\right)}{\sqrt{1+x_{\varphi}^{2}}}-\sqrt{\beta_{\phi} y_{\phi}} x_{\phi}+\gamma\right) \\
x_{\varphi}^{\prime}=-3\left(x_{\varphi}+\sqrt{\beta_{\varphi} y_{\varphi}}\right)\left(1+x_{\varphi}^{2}\right) \\
y_{\varphi}^{\prime}=3 y_{\varphi}\left(-\frac{y_{\phi}\left(\gamma-x_{\phi}^{2}\right)}{\sqrt{1-x_{\phi}^{2}}}-\frac{y_{\varphi}\left(\gamma+x_{\varphi}^{2}\right)}{\sqrt{1+x_{\varphi}^{2}}}-\sqrt{\beta_{\varphi} y_{\varphi}} x_{\varphi}+\gamma\right)
\end{gathered}
$$

where

$$
\beta_{\phi}=\frac{4 M_{p}^{2}}{3 M_{\phi}^{2}}, \quad \beta_{\varphi}=\frac{4 M_{p}^{2}}{3 M_{\varphi}^{2}}
$$

The equation of state of the dark energy is

$$
\omega=\frac{-V(\phi) \sqrt{1-\dot{\phi}^{2}}-V(\varphi) \sqrt{1+\dot{\varphi}^{2}}}{\frac{V(\phi)}{\sqrt{1-\dot{\phi}^{2}}}+\frac{V(\varphi)}{\sqrt{1+\dot{\varphi}^{2}}}}=\frac{-y_{\phi} \sqrt{1-x_{\phi}^{2}}-y_{\varphi} \sqrt{1+x_{\varphi}^{2}}}{\frac{y_{\phi}}{\sqrt{1-x_{\phi}^{2}}}+\frac{y_{\varphi}}{\sqrt{1+x_{\varphi}^{2}}}}
$$



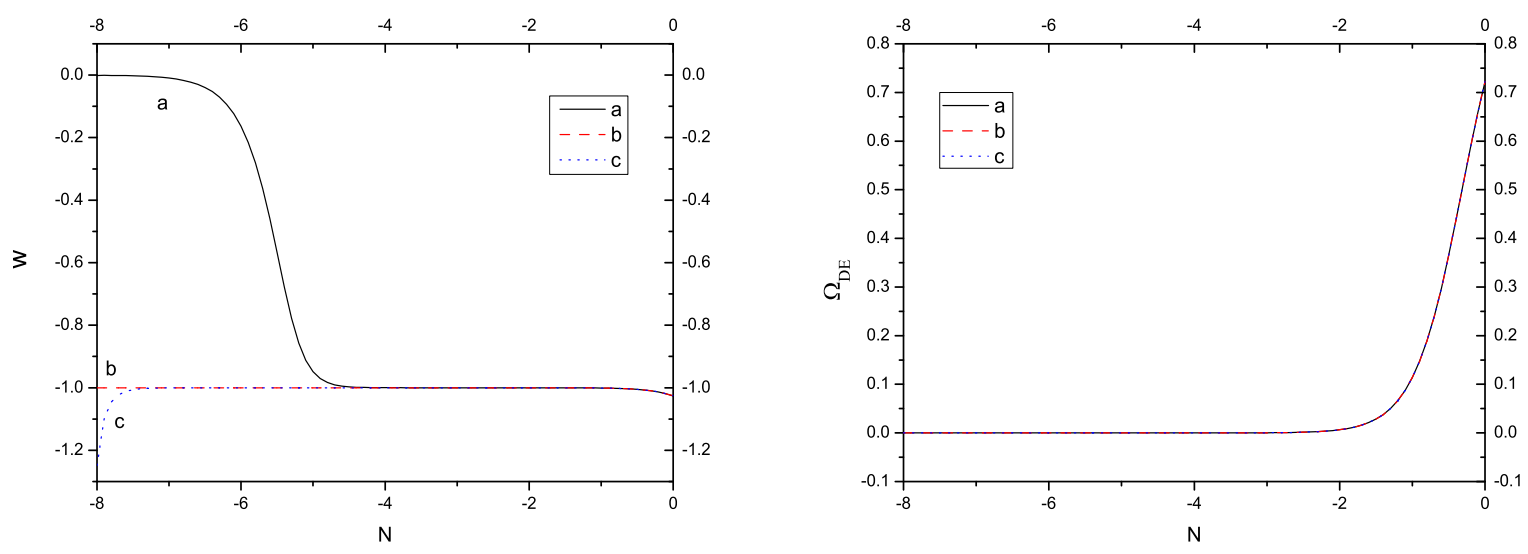

FIG. 1: Evolution of the equation of state $(\omega)$ and density parameters $\left(\Omega_{D E}\right)$ as a function of $\mathrm{N}$ for the dark energy model with $\beta_{\phi}=\beta_{\varphi}=1 / 3, \gamma=1$. Initial conditions $($ at $N=-8)$ : a. solid line: $x_{\phi i}=0.9999999, y_{\phi i}=6 \times 10^{-11}, x_{\varphi i}=2.0, y_{\varphi i}=6.5 \times 10^{-11} ;$ b. dashed line: $x_{\phi i}=1 \times 10^{-12}, y_{\phi i}=6 \times 10^{-11}, x_{\varphi i}=1 \times 10^{-12}, y_{\varphi i}=6.5 \times 10^{-11} ;$ c. dotted line: $x_{\phi i}=$ $0.5, y_{\phi i}=6 \times 10^{-11}, x_{\varphi i}=1.0, y_{\varphi i}=6.5 \times 10^{-11}$.

\section{B. Numerical analysis}

Mapping between the number of $e$-foldings and the redshift $z \equiv a_{0} / a-1=1 / a-1$, we note that at big bang nucleosynthesis $(\mathrm{BBN}) N_{\mathrm{BBN}} \approx-20\left(z \approx 10^{9}\right)$, at matter-radiation equality $N_{\text {eq }} \approx-8(z \approx 3200)$. We choose $N=-8$ as the initial number of e-folds, so choose the $\gamma=1 \mathrm{in} \mathrm{Eqs.} \mathrm{(16)} \mathrm{and} \mathrm{(18)} \mathrm{is} \mathrm{a} \mathrm{good} \mathrm{approximation.} \mathrm{The} \mathrm{evolutions} \mathrm{of} \omega$ and $\Omega_{D E}$ are shown in Fig. 1. In Ref. [28], the authors use standard Big Bang Nucleosynthesis and the observed abundances of primordial nuclides to give a constraints on the scalar matter : $\Omega_{D E}<0.045$, at temperatures near $1 \mathrm{MeV}$. The initial values of $x_{\phi}, y_{\phi}, x_{\varphi}$ and $y_{\varphi}$ given below are safely satisfy this requirement, since $\Omega_{D E} \leq 6 \times 10^{-7}$ at $N=-8$, and the energy density of $\phi$ and $\varphi$ are decreasing more slowly than the fluid $\left(\omega_{\phi}=\frac{p_{\phi}}{\rho_{\phi}}=-1+\dot{\phi}^{2}<0, \omega_{\varphi}=\frac{p_{\varphi}}{\rho_{\varphi}}=-1-\dot{\varphi}^{2} \leq-1\right)$. So at temperatures near $1 \mathrm{MeV}$, the $\Omega_{D E}$ is smaller.

From pictures we can see that this model is not sensitive to the initial kinetic energy density of tachyon and phantom tachyon $\left(x_{\phi}=\dot{\phi}, x_{\varphi}=\dot{\varphi}\right)$. When $x_{\phi i}=0.9999999, y_{\phi i}=$ $6 \times 10^{-11}, x_{\varphi i}=2.0, y_{\varphi i}=6.5 \times 10^{-11}$, the current $\omega$ and $\Omega_{D E}$ are $-1.025896,0.722306$, respectively. When $x_{\phi i}=1 \times 10^{-12}, y_{\phi i}=6 \times 10^{-11}, x_{\varphi i}=1 \times 10^{-12}, y_{\varphi i}=6.5 \times 10^{-11}$, the current $\omega$ and $\Omega_{D E}$ are $-1.025841,0.722367$, respectively. From Eq. (4), we know that the initial energy density of the tachyon varied by nearly four orders of magnitude is still consistent with current observational constraints. But the initial potential energy density of tachyon and phantom tachyon require fine-tuning to agree with observations.

At the present, we want to explain in rough detail how solutions converge to the common 

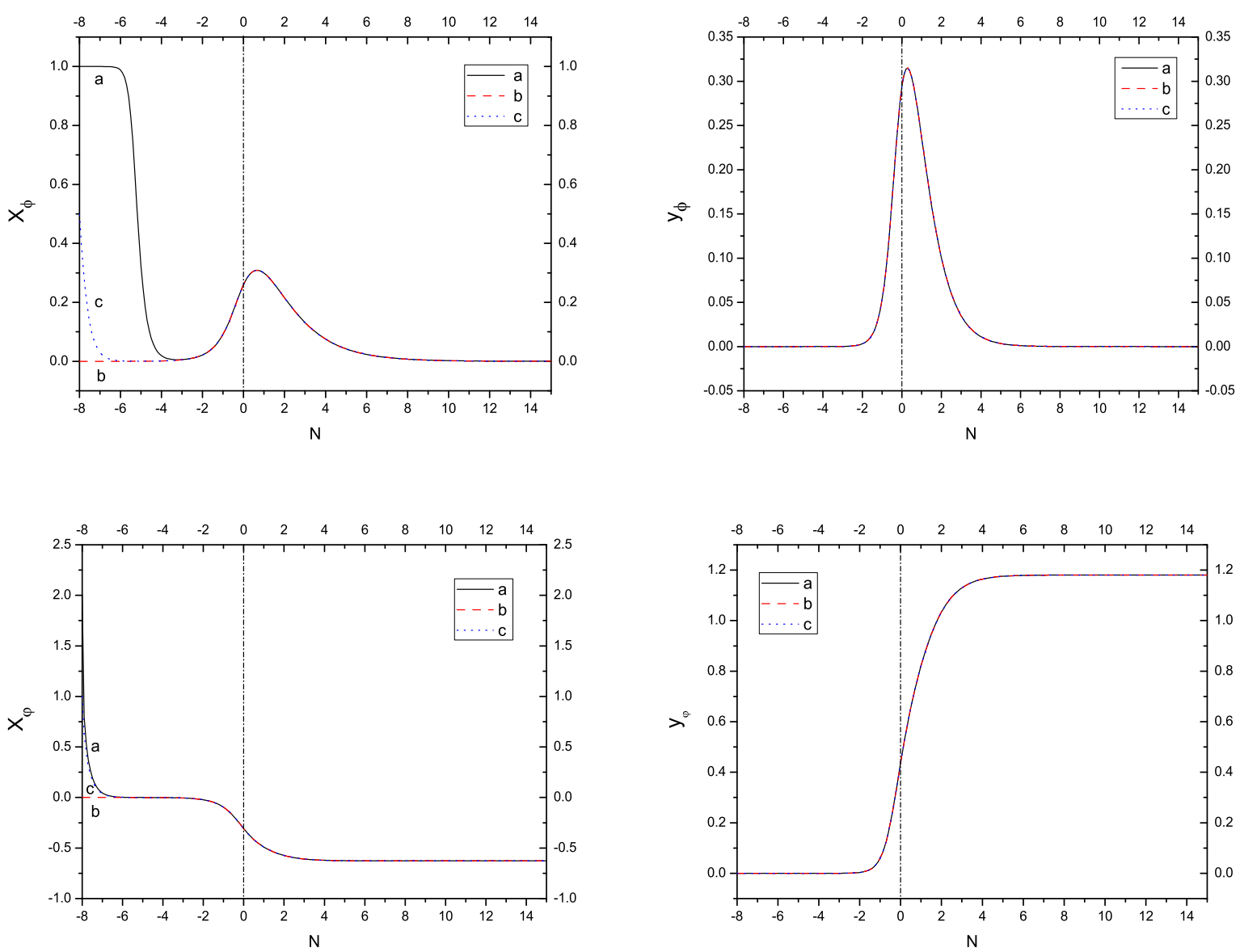

FIG. 2: Evolution of the $x_{\phi}, y_{\phi}, x_{\varphi}$ and $y_{\varphi}$ as a function of $\mathrm{N}$ for the dark energy model with $\beta_{\phi}=\beta_{\varphi}=1 / 3, \gamma=1$. Initial conditions (at $N=-8$ ) : a. solid line: $x_{\phi i}=0.9999999, y_{\phi i}=$ $6 \times 10^{-11}, x_{\varphi i}=2.0, y_{\varphi i}=6.5 \times 10^{-11} ; \quad$ b. dashed line: $x_{\phi i}=1 \times 10^{-12}, y_{\phi i}=6 \times 10^{-11}, x_{\varphi i}=$ $1 \times 10^{-12}, y_{\varphi i}=6.5 \times 10^{-11} ;$ c. dotted line: $x_{\phi i}=0.5, y_{\phi i}=6 \times 10^{-11}, x_{\varphi i}=1.0, y_{\varphi i}=6.5 \times 10^{-11}$.

solution for the different initial conditions which given in Fig.1. (In next subsection, we will know that there is only one stable critical point, so the solutions converge to a common, cosmic evolutionary track is not surprised. )

From the Fig. 2, we can see that the evolution of the $x_{\phi}, y_{\phi}, x_{\varphi}$ and $y_{\varphi}$ as a function of $\mathrm{N}$. We observe that the $y_{\phi}$ and $y_{\varphi}$ evolutionary tracks are not sensitive to the initial conditions of $x_{\phi}$ and $x_{\varphi}$ which given in Fig.11. This can be seen from the Eqs. (16) and (18). The initial value of $x_{\phi}, y_{\phi}, x_{\varphi}$ and $y_{\varphi}$ are so small that we can safely neglect the first three term of the right hand side of Eqs. (16) and (18). So when $-8<N<-2$, we get $y_{\phi}^{\prime} \approx 3 \gamma y_{\phi}, y_{\varphi}^{\prime} \approx 3 \gamma y_{\varphi}$. When $N \geq-2$, the different initial value of $x_{\phi}$ and $x_{\varphi}$ have converged to a common evolutionary track, so from then on even the value of $x_{\phi}, y_{\phi}, x_{\varphi}$ and $y_{\varphi}$ become large, the convergence of $y_{\phi}$ and $y_{\varphi}$ with different initial value of $x_{\phi}$ and $x_{\varphi}$ is no 
change.

Now, consider the case (a) in which the initial value of $x_{\phi a}$ is large, such as the solid line in Fig.2 . Since $x_{\phi a}>\sqrt{\beta_{\phi} y_{\phi a}}$, so $x_{\phi a}$ will decrease until $x_{\phi a}=\sqrt{\beta_{\phi} y_{\phi a}} \equiv x_{A}$ (see Eq. (15) ). Next, consider the case (b) in which the initial value of $x_{\phi b}$ is small, such as the dashed line in Fig.2 . Since $x_{\phi b}<\sqrt{\beta_{\phi} y_{\phi b}}$, so $x_{\phi b}$ will increase until $x_{\phi b}=\sqrt{\beta_{\phi} y_{\phi b}} \equiv x_{B}$. We have observed above that the $y_{\phi}$ evolutionary tracks is not sensitive to the initial value of $x_{\phi}$ in these two case. But this doesn't mean that $x_{A}=x_{B}$, since the corresponding $\mathrm{N}$ might be different, and $y_{\phi}$ vary with $\mathrm{N}$. In order to show these two case will converge, we suppose that $x_{\phi a}=x_{\phi b}+\delta$ at certain point $\mathrm{N}$ (e.g. $N=-4$ ). Since $x_{a \phi}, x_{\phi b}$ are very small, and in some sense $y_{\phi}$ evolutionary track is independent, so the Eq. (15) can be expanded to lowest order in $\delta: \delta^{\prime}=-3 \delta$. The solution of this equation is $\delta \propto e^{-3 N}$. This means that $\delta$ dacays exponentially, and the evolutionary track of different initial value of $x_{\phi}$ given in Fig.2 converge.

Similar analysis can apply to $x_{\varphi}$. So we have proved that the evolutionary tracks of the $x_{\phi}, y_{\phi}, x_{\varphi}$ and $y_{\varphi}$ of different initial value given in Fig.1 are converge.

\section{The future of the universe}

The critical points correspond to the fixed points where $x_{\phi}^{\prime}=0, y_{\phi}^{\prime}=0, x_{\varphi}^{\prime}=0, y_{\varphi}^{\prime}=0$, which have been calculated and given in Table I, and there are self-similar solutions with

$$
\frac{\dot{H}}{H^{2}}=-\frac{3}{2}\left(-\frac{y_{\phi}\left(\gamma-x_{\phi}^{2}\right)}{\sqrt{1-x_{\phi}^{2}}}-\frac{y_{\varphi}\left(\gamma+x_{\varphi}^{2}\right)}{\sqrt{1+x_{\varphi}^{2}}}+\gamma\right)
$$

This corresponds to an expanding universe with a scale factor $a(t)$ given by $a \propto t^{p}$, where

$$
p=\frac{2}{3\left(-\frac{y_{\phi}\left(\gamma-x_{\phi}^{2}\right)}{\sqrt{1-x_{\phi}^{2}}}-\frac{y_{\varphi}\left(\gamma+x_{\varphi}^{2}\right)}{\sqrt{1+x_{\varphi}^{2}}}+\gamma\right)}
$$

We now study the stability around the critical points given in Table I. Consider small perturbations $\delta x_{\phi}, \delta y_{\phi}, \delta x_{\varphi}$, and $\delta x_{\varphi}$ about the critical points $\left(x_{\phi c}, y_{\phi c}, x_{\varphi c}, y_{\varphi c}\right): x_{\phi c} \rightarrow$ $x_{\phi c}+\delta x_{\phi}, y_{\phi c} \rightarrow y_{\phi c}+\delta y_{\phi}, x_{\varphi c} \rightarrow x_{\varphi c}+\delta x_{\varphi}, y_{\varphi c} \rightarrow y_{\varphi c}+\delta y_{\varphi}$.

Substituting into Eqs. (15) -(18) , lead to the first-order differential equations:

$$
\left(\begin{array}{c}
\delta x_{\phi}^{\prime} \\
\delta y_{\phi}^{\prime} \\
\delta x_{\varphi}^{\prime} \\
\delta y_{\varphi}^{\prime}
\end{array}\right)=\mathcal{M}\left(\begin{array}{l}
\delta x_{\phi} \\
\delta y_{\phi} \\
\delta x_{\varphi} \\
\delta y_{\varphi}
\end{array}\right)
$$

where $\mathcal{M}$ is a matrix that depends upon $x_{\phi c}, y_{\phi c}, x_{\varphi c}$ and $y_{\varphi c}$. 


\begin{tabular}{cccccc}
\hline Label & $x_{\phi c}$ & $y_{\phi c}$ & $x_{\varphi c}$ & $y_{\varphi c}$ & Existence \\
\hline$A$. & 0 & 0 & 0 & 0 & all $\gamma$ \\
$B$. & 0 & 0 & $-\sqrt{\beta_{\varphi} y_{\varphi c}}$ & $\frac{\sqrt{\beta_{\varphi}^{2}+4}+\beta_{\varphi}}{2}$ & all $\gamma$ \\
$C$. & \pm 1 & 0 & 0 & 0 & all $\gamma$ \\
$D$. & \pm 1 & 0 & $-\sqrt{\beta_{\varphi} y_{\varphi c}}$ & $\frac{\sqrt{\beta_{\varphi}^{2}+4}+\beta_{\varphi}}{2}$ & all $\gamma$ \\
$E$. & 1 & $\frac{1}{\beta_{\phi}}$ & 0 & 0 & $\gamma=1$ \\
$F$. & -1 & $\frac{\beta_{\varphi}^{2} y_{\varphi c}^{2}}{\beta_{\phi}}$ & $-\sqrt{\beta_{\varphi} y_{\varphi c}}$ & $\frac{\sqrt{\beta_{\varphi}^{2}+4}+\beta_{\varphi}}{2}$ & $\gamma=1$ \\
$G$. & $\sqrt{\gamma}$ & $\frac{\gamma}{\beta_{\phi}}$ & 0 & 0 & $\gamma<\frac{1}{2}\left(\beta_{\phi} \sqrt{\beta_{\phi}^{2}+4}-\beta_{\phi}^{2}\right)$ \\
$H$. & $\sqrt{\beta_{\phi} y_{\phi c}}$ & $\frac{\sqrt{\beta_{\phi}^{2}+4}-\beta_{\phi}}{2}$ & 0 & 0 & all $\gamma$ \\
& & & & & \\
\hline
\end{tabular}

TABLE I: The list of the critical points.

\begin{tabular}{cccccc}
\hline Label & $m_{1}$ & $m_{2}$ & $m_{3}$ & $m_{4}$ & Stability \\
\hline$A$. & -3 & $3 \gamma$ & -3 & $3 \gamma$ & unstable \\
$B$. & -3 & $-3 x_{\varphi_{c}}^{2}$ & $-\frac{3}{2}\left(2+x_{\varphi c}^{2}\right)$ & $-3\left(\gamma+x_{\varphi c}^{2}\right)$ & stable \\
$C$. & 6 & $3 \gamma$ & -3 & $3 \gamma$ & unstable \\
$D$. & 6 & $-3 x_{\varphi c}^{2}$ & $-\frac{3}{2}\left(2+x_{\varphi c}^{2}\right)$ & $-3\left(\gamma+x_{\varphi c}^{2}\right)$ & unstable \\
$E$. & 0 & $-\frac{3}{2}$ & -3 & 3 & unstable \\
$F$. & $6+6 x_{\varphi c}^{2}$ & $\frac{3}{2} x_{\varphi c}^{2}$ & $-\frac{3}{2}\left(2+x_{\varphi c}^{2}\right)$ & $-3\left(\gamma+x_{\varphi c}^{2}\right)$ & unstable \\
$G$. & $a$ & $b$ & -3 & $3 \gamma$ & unstable \\
$H$. & $-3 \gamma+3 x_{\phi c}^{2}$ & $-3+\frac{3 x_{\phi c}^{2}}{2}$ & -3 & $3 x_{\phi c}^{2}$ & unstable \\
\hline
\end{tabular}

where: $\quad a, b=\frac{1}{4 \beta_{\phi}}\left(\beta_{\phi}(3 \gamma-6) \pm 3 \sqrt{16 \beta_{\phi} \gamma^{2} \sqrt{1-\gamma}+\beta_{\phi}^{2}\left(4-20 \gamma+17 \gamma^{2}\right)}\right)$

TABLE II: The eigenvalues and stability of the critical points.

The general solution for the evolution of linear perturbations can be written as

$$
\begin{aligned}
& \delta x_{\phi}=u_{11} \exp \left(m_{1} N\right)+u_{12} \exp \left(m_{2} N\right)+u_{13} \exp \left(m_{3} N\right)+u_{14} \exp \left(m_{4} N\right) \\
& \delta y_{\phi}=u_{21} \exp \left(m_{1} N\right)+u_{22} \exp \left(m_{2} N\right)+u_{23} \exp \left(m_{3} N\right)+u_{24} \exp \left(m_{4} N\right) \\
& \delta x_{\varphi}=u_{31} \exp \left(m_{1} N\right)+u_{32} \exp \left(m_{2} N\right)+u_{33} \exp \left(m_{3} N\right)+u_{34} \exp \left(m_{4} N\right) \\
& \delta y_{\varphi}=u_{41} \exp \left(m_{1} N\right)+u_{42} \exp \left(m_{2} N\right)+u_{43} \exp \left(m_{3} N\right)+u_{44} \exp \left(m_{4} N\right)
\end{aligned}
$$

where $m_{1}, m_{2}, m_{3}$, and $m_{4}$ are the eigenvalues of the matrix $\mathcal{M}$. Thus stability requires 

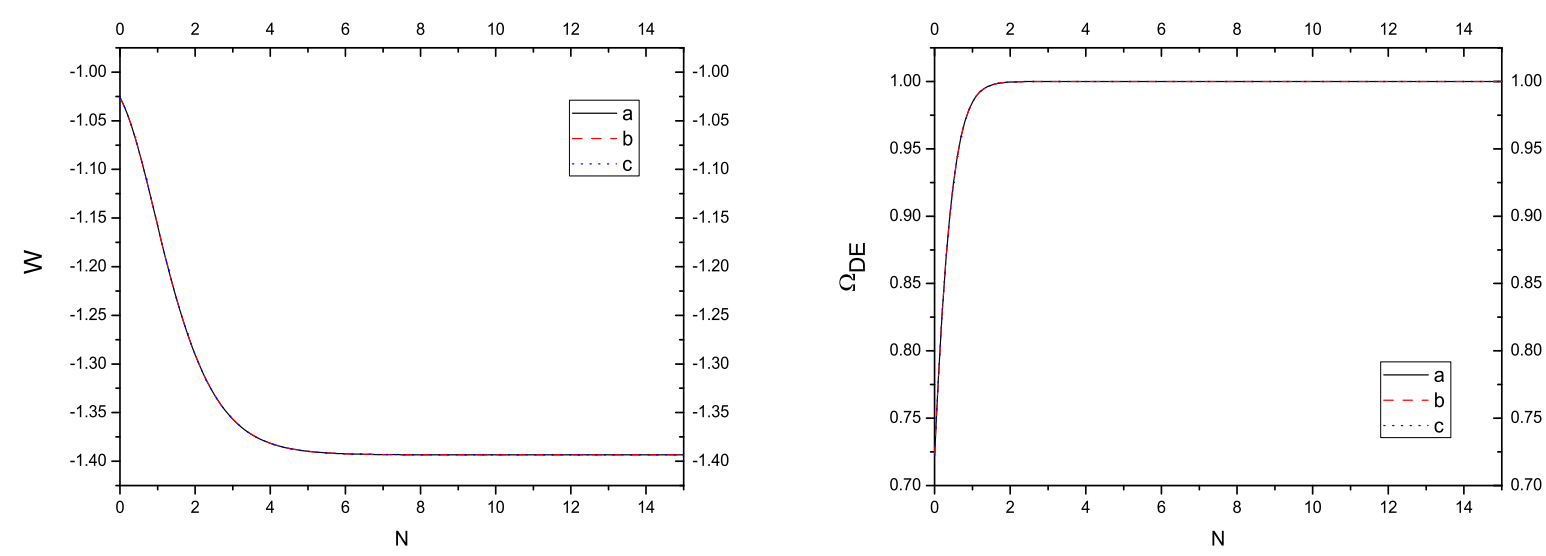

FIG. 3: Evolution of the equation of state $(\omega)$ and density parameters $\left(\Omega_{D E}\right)$ as a function of $\mathrm{N}$ for the dark energy model with $\beta_{\phi}=\beta_{\varphi}=1 / 3, \gamma=1$. Initial conditions $($ at $N=-8)$ : a. solid line: $x_{\phi i}=0.9999999, y_{\phi i}=6 \times 10^{-11}, x_{\varphi i}=2.0, y_{\varphi i}=6.5 \times 10^{-11} ;$ b. dashed line: $x_{\phi i}=1 \times 10^{-12}, y_{\phi i}=6 \times 10^{-11}, x_{\varphi i}=1 \times 10^{-12}, y_{\varphi i}=6.5 \times 10^{-11} ;$ c. dotted line: $x_{\phi i}=$ $0.5, y_{\phi i}=6 \times 10^{-11}, x_{\varphi i}=1.0, y_{\varphi i}=6.5 \times 10^{-11}$.

the real part of all eigenvalues being negatives [26, 29].

We obtain the eigenvalues and stability for the fixed points in Table II. The system has a fixed point $\mathrm{A}$ which is a fluid-dominated solution, a fixed point $\mathrm{B}$ which is a phantom tachyon-dominated solution, a fixed point $\mathrm{C}$ which is tachyon kinetic-dominated solution, a fixed point $\mathrm{D}$ which is the two-field dominated solution, two fixed points $\mathrm{E}$ and $\mathrm{F}$ which exist only for $\gamma=1$, a fixed point $\mathrm{G}$ in which the energy densities $\rho_{\phi}$ and $\rho_{\gamma}$ decrease with the same rate, a fixed $\mathrm{H}$ which is tachyon dominated solution.

In Fig. 3, we plot the evolution of the equation of state in the future. We find that the cosmic evolutionary track is towards the stable fixed point B in this model. This can be seen by compare Table I with Fig.2:

Substituting $\beta_{\varphi}=1 / 3$ into the stable fixed point B $\left(x_{\phi c}=0, y_{\phi c}=0, x_{\varphi c}=-\sqrt{\beta_{\varphi} y_{\varphi c}}\right.$, $\left.y_{\varphi c}=\left(\sqrt{\beta_{\varphi}^{2}+4}+\beta_{\varphi}\right) / 2\right)$, we get $x_{\phi c}=0, y_{\phi c}=0, x_{\varphi c}=-0.627285, y_{\varphi c}=1.180460$, which are consistent with Fig.2. And from Eq. (20), we know $\omega=-\left(1+x_{\varphi c}^{2}\right)=-1.393487$ at the fix point B, which is consistent with Fig. 3.

Generally speaking, if the initial values of $x_{\phi}, y_{\phi}, x_{\varphi}$ and $y_{\varphi}$ are not the values of the unstable critical point given in Table I, they will evolve towards the stable critical point $\mathrm{B}$ ( If the physical constraints $1-x_{\phi}^{2}>0$ is not violated ). This point can be seen from Eq. (24). (If one evolutionary track is towards the unstable critical point, when the values of $x_{\phi}, y_{\phi}, x_{\varphi}$ and $y_{\varphi}$ differ from $x_{\phi c}, y_{\phi c}, x_{\varphi c}, y_{\varphi c}$ by an amount $\vec{\delta}$, then from Eq. (24), we know the $\vec{\delta}$ will be larger, instead of becoming smaller. ) 


\section{Discussions}

- In a tachyon dark energy model, the tachyon is the only source of the dark energy, and there are three kind stable critical points [22], which existence depend on the value of $\gamma$. But in our model, there is only one stable critical point, which existence is independent of the value of $\gamma$. In a tachyon dark energy model, the value of $x_{\phi}$ and $y_{\phi}$ can be non-zero at the stable critical points. But in our model the value of $x_{\phi}$ and $y_{\phi}$ must be zero, which we have shown in table I. This's not accident. This point can be seen as follows. At the critical point, the value of $x_{\phi c}, y_{\phi c}, x_{\varphi c}$ and $y_{\varphi c}$ are fixed, and the value of $x_{\varphi c}$ is non-zero, otherwise the value of $y_{\varphi c}$ is zero (see Eq. (17)), which means $\rho_{\varphi c}=0$, so $x_{\varphi c}=0$ is impossible.

$$
\begin{aligned}
\rho_{\phi c}=\frac{V\left(\phi_{c}\right)}{\sqrt{1-\dot{\phi}_{c}^{2}}}=\frac{3 M_{p}^{2} y_{\phi c}}{\sqrt{1-x_{\phi c}^{2}}} H^{2} & \omega_{\phi c}=\frac{p_{\phi c}}{\rho_{\phi c}}=-1+{\dot{\phi_{c}}}^{2}=-1+x_{\phi c}^{2} \geq-1 \\
\rho_{\varphi c}=\frac{V\left(\varphi_{c}\right)}{\sqrt{1-\dot{\varphi}_{c}^{2}}}=\frac{3 M_{p}^{2} y_{\varphi c}}{\sqrt{1-x_{\varphi c}^{2}}} H^{2} & \omega_{\varphi c}=\frac{p_{\varphi c}}{\rho_{\varphi c}}=-1-\dot{\varphi}_{c}^{2}=-1-x_{\varphi c}^{2}<-1
\end{aligned}
$$

If $y_{\phi c} \neq 0$, then from Eq. (25), we know $H^{2}$ is nonincreasing at the fix points, since $\rho_{\phi c}$ is nonincreasing. If $y_{\varphi c} \neq 0$, then from Eq. (26), we know $H^{2}$ is increasing at the fix points, since $\rho_{\varphi c}$ is increasing. So either $y_{\phi c}$ or $y_{\varphi c}$ will be zero. Since $\rho_{\varphi c}$ is increasing and $\rho_{\phi c}$ is nonincreasing, so we choose $y_{\phi c}=0$. And from Eq. (15), we know $x_{\phi c}=0$. (This is because $y_{\phi c}=0$, and if the physical constraints $1-x_{\phi c}^{2}>0$ is not violated .)

- In a tachyon dark energy model with inverse square potential $V(\phi)=M_{\phi}^{2} \phi^{-2}$, in order to have significant acceleration at late times $\left(a \propto t^{p}, p \equiv \frac{1}{2}\left(\frac{M_{\phi}}{M_{p}}\right)^{2} \gg 1\right)$, we clearly require $M_{\phi}$ much larger than the Planck mass [23]. Such a large mass is problematic as we expect general relativity itself to break down in such a regime. This problem is fortunately alleviated for the inverse power-law potential $V=M_{\phi}^{4-n} \phi^{-n}$ with $0<n<2$. In our model, since there is another field $\varphi$, with the equation of state $\omega_{\varphi}<-1$, a significant acceleration at late times can be obtained much easier. But the value of $M_{\phi}$ still can't be small very much, since if $\beta_{\phi}=\frac{4 M_{p}^{2}}{3 M_{\phi}^{2}}$ is larger, the risk of $1-\dot{\phi}^{2}$ becoming nonpositive is increasing, which can be see from Eqs. (15) and (16).

- The speed of sound describe the evolution of small perturbations. In a tachyon dark energy model the sound speed is

$$
c_{s}^{2}=\frac{p_{\phi X}}{\rho_{\phi X}}=1-\dot{\phi}^{2}
$$

where $X$ denotes the partial derivative with respect to $X=\frac{1}{2}\left(\partial_{\mu} \phi\right)^{2}$. Since the value of $1-\dot{\phi}^{2}$ is necessarily nonpositive because of the square root in the Lagrangian density Eq. (21), the energy and pressure are real, and inhomogeneous perturbations have a 
positive sound speed, so the theory is stable. In our model, there are two fields. Physically we can use the independent sound speed of each component to describe the whole system. However, the present constraints on the sound speed of dark energy are so weak that considering to study the two independent sound speed is not justified at present. So it can use the effective sound speed, as some authors do [30, 31]. As we have shown above, when $\mathrm{N}$ is large enough, the fractional energy density of phantom tachyon $\Omega_{\varphi} \rightarrow 1$. So ultimately the effective Lagrangian density is Eq. (3), and the effective sound speed is

$$
c_{s}^{2}=\frac{p_{\varphi X}}{\rho_{\varphi X}}=1+\dot{\varphi}^{2}>1
$$

This means that perturbations of the background scalar field can travel faster than light as measured in the preferred frame where the background field is homogeneous. But there is no violation of causality. The theory of the k-essence-like scalar fields with the Lorentz invariant action is not possible create closed time-like curves in the Friedmann universe and hence we cannot send the signal to our own past using the superluminal signals build out of the superluminal scalar field perturbations [32].

\section{ANOTHER TACHYON-QUINTOM MODEL INCLUDING THE INTERACTION BETWEEN TWO FIELDS}

In order to show some impact of interactions between the two scalars on the evolution of the universe, we consider another system, which include a fluid with barotropic equation of state $p_{\gamma}=(\gamma-1) \rho_{\gamma}, 0<\gamma \leq 2$, and two scalars with interaction between them. Maybe there are many more interactions between the two scalars, but for simplicity we only consider the below Lagrangian density, to see whether there are some interesting results or not.

The Lagrangian density of the scalars we choose are:

$$
\mathcal{L}=-V(\phi, \varphi) \sqrt{1+g^{\mu \nu} \partial_{\mu} \phi \partial_{\nu} \phi-g^{\mu \nu} \partial_{\mu} \varphi \partial_{\nu} \varphi}
$$

In this section, we turn our attention to the possibility of the scalars as a source of the dark energy. We restrict to spatially homogeneous time dependent solutions for which $\partial_{i} \phi=\partial_{i} \varphi=0$. Thus the energy densities and the pressure of the fields are

$$
\rho=\frac{V(\phi, \varphi)}{\sqrt{1-\dot{\phi}^{2}+\dot{\varphi}^{2}}}, \quad p=-V(\phi, \varphi) \sqrt{1-\dot{\phi}^{2}+\dot{\varphi}^{2}}
$$

Here a dot is derivation with respect to synchronous time. The background equations of motion are

$$
\begin{aligned}
& \frac{\ddot{\phi}+\ddot{\phi} \dot{\varphi}^{2}-\ddot{\varphi} \dot{\phi} \dot{\varphi}}{1-\dot{\phi}^{2}+\dot{\varphi}^{2}}+3 H \dot{\phi}+\left(1+\dot{\varphi}^{2}\right) \frac{d V(\phi)}{V d \phi}=0 \\
& \frac{\ddot{\varphi}-\ddot{\varphi} \dot{\phi}^{2}+\ddot{\phi} \dot{\phi} \dot{\varphi}}{1-\dot{\phi}^{2}+\dot{\varphi}^{2}}+3 H \dot{\varphi}-\left(1-\dot{\phi}^{2}\right) \frac{d V(\varphi)}{V d \varphi}=0
\end{aligned}
$$




$$
\begin{gathered}
\dot{\rho}_{\gamma}=-3 \gamma H \rho_{\gamma} \\
\dot{H}=-\frac{1}{2 M_{p}^{2}}\left(\frac{\dot{\phi}^{2} V(\phi, \varphi)}{\sqrt{1-\dot{\phi}^{2}+\dot{\varphi}^{2}}}-\frac{\dot{\varphi}^{2} V(\phi, \varphi)}{\sqrt{1-\dot{\phi}^{2}+\dot{\varphi}^{2}}}+\gamma \rho_{\gamma}\right)
\end{gathered}
$$

together with a constraint equation for the Hubble parameter:

$$
H^{2}=\frac{1}{3 M_{p}^{2}}\left(\frac{V(\phi, \varphi)}{\sqrt{1-\dot{\phi}^{2}+\dot{\varphi}^{2}}}+\rho_{\gamma}\right)
$$

The potentials we considered are still inverse square potentials:

$$
V(\phi, \varphi)=\lambda_{1} M_{p}^{2} \phi^{-1} \varphi^{-1}+\lambda_{2} M_{p}^{2} \phi^{-2}+\lambda_{3} M_{p}^{2} \varphi^{-2}
$$

We define the following dimensionless quantities :

$$
x_{\phi}=\dot{\phi}, y_{\phi}=\frac{\phi^{-1}}{\sqrt{3} H}, X_{\varphi}=\dot{\varphi}, y_{\varphi}=\frac{\varphi^{-1}}{\sqrt{3} H}, z=\frac{\rho_{\gamma}}{3 H^{2} M_{p}^{2}}
$$

Now the Eqs. (35) and (34) can be rewrite as follow:

$$
\begin{gathered}
1=\frac{\lambda_{1} y_{\phi} y_{\varphi}+\lambda_{2} y_{\phi}^{2}+\lambda_{3} y_{\varphi}^{2}}{\sqrt{1-x_{\phi}^{2}+x_{\varphi}^{2}}}+z=\Omega_{D E}+z \\
\frac{H^{\prime}}{H}=-\frac{3}{2}\left[\frac{-\lambda_{1} y_{\phi} y_{\varphi}\left(\gamma-x_{\phi}^{2}+x_{\varphi}^{2}\right)-\lambda_{2} y_{\phi}^{2}\left(\gamma-x_{\phi}^{2}+x_{\varphi}^{2}\right)-\lambda_{3} y_{\varphi}^{2}\left(\gamma-x_{\phi}^{2}+x_{\varphi}^{2}\right)}{\sqrt{1-x_{\phi}^{2}+x_{\varphi}^{2}}}+\gamma\right]
\end{gathered}
$$

where $\Omega_{D E}$ measure the dark energy density as a fraction of the critical density, a prime denotes a derivative with respect to the logarithm of the scale factor, $N=\ln a$.

Then the evolution Eqs. (31) and (32) can be written to an autonomous system:

$$
\begin{gathered}
x_{\phi}^{\prime}=-3\left(1-x_{\phi}^{2}\right)\left[x_{\phi}+\left(\frac{-\sqrt{3} \lambda_{1} y_{\phi}-2 \sqrt{3} \lambda_{2} y_{\phi}^{2} / y_{\varphi}}{3 \lambda_{1}+3 \lambda_{2} y_{\phi} / y_{\varphi}+3 \lambda_{3} y_{\varphi} / y_{\phi}}\right)\left(1+x_{\varphi}^{2}\right)\right] \\
-3 x_{\phi} x_{\varphi}\left[x_{\varphi}-\left(\frac{-\sqrt{3} \lambda_{1} y_{\varphi}-2 \sqrt{3} \lambda_{3} y_{\varphi}^{2} / y_{\phi}}{3 \lambda_{1}+3 \lambda_{2} y_{\phi} / y_{\varphi}+3 \lambda_{3} y_{\varphi} y_{\phi}}\right)\left(1-x_{\phi}^{2}\right)\right] \\
y_{\phi}^{\prime}=y_{\phi}\left[\frac{-3 \lambda_{1} y_{\phi} y_{\varphi}\left(\gamma-x_{\phi}^{2}+x_{\varphi}^{2}\right)-3 \lambda_{2} y_{\phi}^{2}\left(\gamma-x_{\phi}^{2}+x_{\varphi}^{2}\right)-3 \lambda_{3} y_{\varphi}^{2}\left(\gamma-x_{\phi}^{2}+x_{\varphi}^{2}\right)}{2 \sqrt{1-x_{\phi}^{2}+x_{\varphi}^{2}}}\right]-\sqrt{3} x_{\phi} y_{\phi}^{2}+\frac{3}{2} \gamma y_{\phi}
\end{gathered}
$$



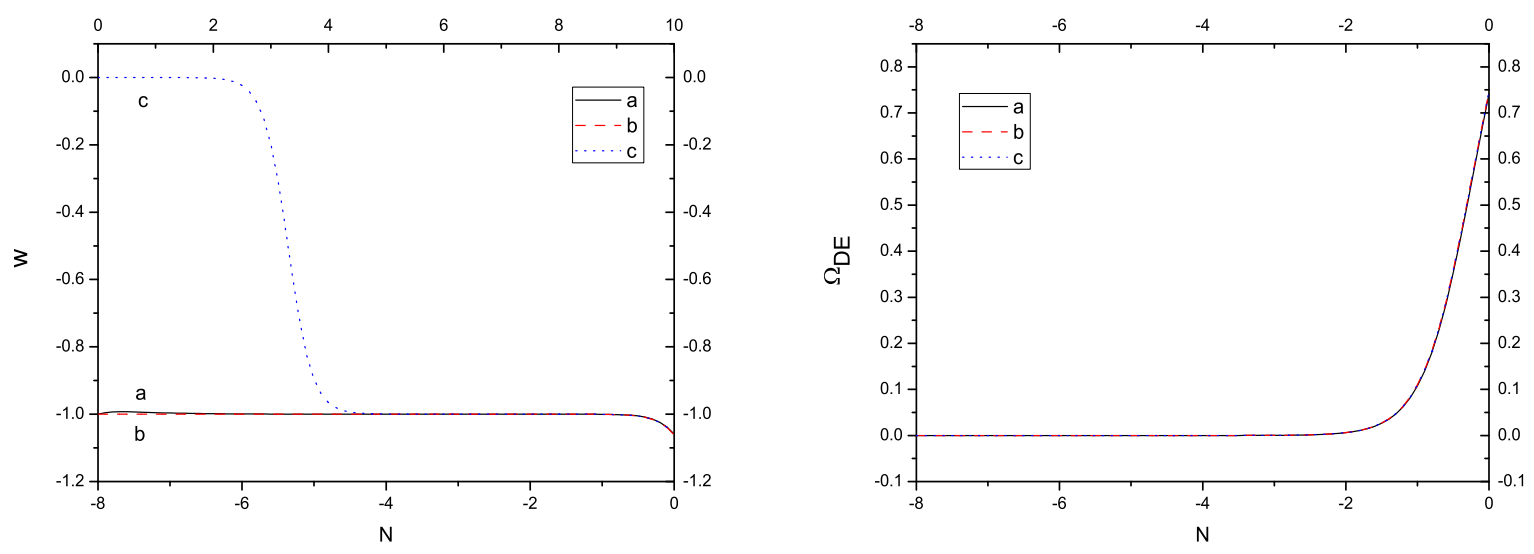

FIG. 4: Evolution of the equation of state $(\omega)$ and density parameters $\left(\Omega_{D E}\right)$ as a function of $\mathrm{N}$ for the dark energy model with $\lambda_{1}=1, \gamma=1$. Initial conditions (at $N=-8$ ) : a. solid line: $x_{\phi i}=1000.0, y_{\phi i}=1 \times 10^{-5}, x_{\varphi i}=1000.0, y_{\varphi i}=1 \times 10^{-5}$; b. dashed line: $x_{\phi i}=1 \times 10^{-12}, y_{\phi i}=$ $1 \times 10^{-5}, x_{\varphi i}=1 \times 10^{-12}, y_{\varphi i}=1 \times 10^{-5} ;$ c. dotted line: $x_{\phi i}=0.9999999, y_{\phi i}=1 \times 10^{-5}, x_{\varphi i}=$ $1 \times 10^{-12}, y_{\varphi i}=1 \times 10^{-5}$.

$$
\begin{gathered}
x_{\varphi}^{\prime}=-3\left(1+x_{\varphi}^{2}\right)\left[x_{\varphi}-\left(\frac{-\sqrt{3} \lambda_{1} y_{\varphi}-2 \sqrt{3} \lambda_{3} y_{\varphi}^{2} / y_{\phi}}{3 \lambda_{1}+3 \lambda_{2} y_{\phi} / y_{\varphi}+3 \lambda_{3} y_{\varphi} / y_{\phi}}\right)\left(1-x_{\phi}^{2}\right)\right] \\
+3 x_{\phi} x_{\varphi}\left[x_{\phi}+\left(\frac{-\sqrt{3} \lambda_{1} y_{\phi}-2 \sqrt{3} \lambda_{2} y_{\phi}^{2} / y_{\varphi}}{3 \lambda_{1}+3 \lambda_{2} y_{\phi} / y_{\varphi}+3 \lambda_{3} y_{\varphi} / y_{\phi}}\right)\left(1+x_{\varphi}^{2}\right)\right] \\
y_{\varphi}^{\prime}=y_{\varphi}\left[\frac{-3 \lambda_{1} y_{\phi} y_{\varphi}\left(\gamma-x_{\phi}^{2}+x_{\varphi}^{2}\right)-3 \lambda_{2} y_{\phi}^{2}\left(\gamma-x_{\phi}^{2}+x_{\varphi}^{2}\right)-3 \lambda_{3} y_{\varphi}^{2}\left(\gamma-x_{\phi}^{2}+x_{\varphi}^{2}\right)}{2 \sqrt{1-x_{\phi}^{2}+x_{\varphi}^{2}}}\right]-\sqrt{3} x_{\varphi} y_{\varphi}^{2}+\frac{3}{2} \gamma y_{\varphi}
\end{gathered}
$$

The equation of state of the dark energy is

$$
\omega=\frac{p}{\rho}=-1+\dot{\phi}^{2}-\dot{\varphi}^{2}=-1+x_{\phi}^{2}-x_{\varphi}^{2}
$$

For simplicity, we confine ourselves to the case $\lambda_{1} \neq 0, \lambda_{2}=\lambda_{3}=0$, then the Eqs. (40)(43) can be reduced to

$$
\begin{gathered}
x_{\phi}^{\prime}=-3\left(1-x_{\phi}^{2}\right)\left(x_{\phi}-\frac{y_{\phi}}{\sqrt{3}}\left(1+x_{\varphi}^{2}\right)\right)-3 x_{\phi} x_{\varphi}\left(x_{\varphi}+\frac{y_{\varphi}}{\sqrt{3}}\left(1-x_{\phi}^{2}\right)\right) \\
y_{\phi}^{\prime}=\frac{-3 \lambda_{1} y_{\phi}^{2} y_{\varphi}\left(\gamma-x_{\phi}^{2}+x_{\varphi}^{2}\right)}{2 \sqrt{1-x_{\phi}^{2}+x_{\varphi}^{2}}}-\sqrt{3} x_{\phi} y_{\phi}^{2}+\frac{3}{2} \gamma y_{\phi} \\
x_{\varphi}^{\prime}=-3\left(1+x_{\varphi}^{2}\right)\left(x_{\varphi}+\frac{y_{\varphi}}{\sqrt{3}}\left(1-x_{\phi}^{2}\right)\right)+3 x_{\phi} x_{\varphi}\left(x_{\phi}-\frac{y_{\phi}}{\sqrt{3}}\left(1+x_{\varphi}^{2}\right)\right)
\end{gathered}
$$




\begin{tabular}{cccccc}
\hline Label & $x_{\phi}$ & $y_{\phi}$ & $x_{\varphi}$ & $y_{\varphi}$ & Existence \\
\hline$A$. & 0 & 0 & 0 & 0 & all $\gamma$ \\
$B$. & $\sqrt{\frac{\gamma}{2}}$ & $\sqrt{\frac{3 \gamma}{2}}$ & 0 & 0 & all $\gamma$ \\
\hline
\end{tabular}

TABLE III: The list of the critical points.

\begin{tabular}{cccccc}
\hline Label & $m_{1}$ & $m_{2}$ & $m_{3}$ & $m_{4}$ & Stability \\
\hline$A$. & -3 & $\frac{3 \gamma}{2}$ & -3 & $\frac{3 \gamma}{2}$ & unstable \\
$B$. & $\frac{1}{2}\left(-3+3 \sqrt{1+2 \gamma^{2}-4 \gamma}\right)$ & $\frac{1}{2}\left(-3-3 \sqrt{1+2 \gamma^{2}-4 \gamma}\right)$ & -3 & $\frac{3 \gamma}{2}$ & unstable \\
\hline
\end{tabular}

TABLE IV: The eigenvalues and stability of the critical points.

$$
y_{\varphi}^{\prime}=\frac{-3 \lambda_{1} y_{\phi} y_{\varphi}^{2}\left(\gamma-x_{\phi}^{2}+x_{\varphi}^{2}\right)}{2 \sqrt{1-x_{\phi}^{2}+x_{\varphi}^{2}}}-\sqrt{3} x_{\varphi} y_{\varphi}^{2}+\frac{3}{2} \gamma y_{\varphi}
$$

The evolution of the equation of state $\omega$ and density parameters are shown in Fig. [4 with $\lambda_{1}=1, \gamma=1$. We choose $N=-8$ as the initial number of e-folds, so choose the $\gamma=1$ in Eqs. (46) and (48) is a good approximation. From Fig.4 we can see that this model is not sensitive to the initial kinetic energy density of the two fields $\left(x_{\phi}=\dot{\phi}, x_{\varphi}=\dot{\varphi}\right)$. From Eq. (30) and Fig.4, we know that the initial energy density of $\phi$ varied by about four orders of magnitude is still consistent with current observational constraints. But the initial potential energy density of the two fields require fine-tuning to agree with observations.

The critical points correspond to the fixed points where $x_{\phi}^{\prime}=0, y_{\phi}^{\prime}=0, x_{\varphi}^{\prime}=0, y_{\varphi}^{\prime}=0$, which have been calculated and given in Table III. To study the stability of the critical points, we substitute the linear perturbations about the critical points into Eq. (45) - (48) and keep terms to the first-order in the perturbations. The four perturbation equations give rise to four eigenvalues. The stability requires the real part of all eigenvalues be negative (see Table IV for the eigenvalues of perturbation equations and the stability of critical points).

So there are no stable critical points. Since the two kind critical points all have $y_{\varphi}=0$, which means that $z=1$ (see Eq. (38)). The physical constraints $1-\dot{\phi}^{2}+\dot{\varphi}^{2}>0$ set limit on the equation of state $\omega$ (see Eq. (44)) $: \omega<0$. Compare this with $\gamma,(\gamma=4 / 3$ for radiation, $\gamma=1$ for dust matter), we know that the energy densities of the fields $\rho$ decrease more slowly than $\rho_{\gamma}$, so $z=1$ and $y_{\varphi}=0$ are impossible.

\section{SUMMARY}

In this paper we have studied the tachyon-quintom dark energy models, in which during the evolution of the universe the equation of state $w$ changes from $w>-1$ to $w<-1$. Firstly, the model we studied is made up of two fields, one is tachyon, the other is phantom 
tachyon. In order to construct a autonomous system, the potentials we choose are inverse square potentials. We find the model is not sensitive to the initial kinetic energy density of tachyon and phantom tachyon, and we analyze the reason in detail. The initial energy density (at $\mathrm{N}=8$ ) of the tachyon varied by nearly four orders of magnitude is still consistent with current observational constraints. The phase-space analysis of the spatially flat FRW models shows that there exist a unique stable critical point, and we compare it with tachyon model at last. Then we consider another form of two-field model which include the interaction between two fields. For the case of $\lambda_{1} \neq 0, \lambda_{2}=0, \lambda_{3}=0$, the phase-space analysis shows that there is no stable critical point. In some sense, this work means that multiple kessencelike fields can implement the quintom, which extends the possibilities that the quintom is realized and is worth further study.

Acknowledgements: This work is supported in part by NSFC under Grant No: 10491306, 10521003, 10775179, 10405029, 10775180, in part by the Scientific Research Fund of GUCAS(NO.055101BM03), in part by CAS under Grant No: KJCX3-SYW-N2.

[1] S. Perlmutter et al., Astrophys. J. 483, 565 (1997); A. G. Riess et al., Astron. J. 116, 1009 (1998); Astron. J. 117, 707 (1999).

[2] D. N. Spergel et al., Astrophys. J. Suppl. 148, 175 (2003).

[3] A. G. Riess et al., Astrophys. J. 607, 665 (2004).

[4] E. J. Copeland, M. Sami, and S. Tsujikawa, Int. J. Mod. Phys. D15,1753 (2006).

[5] I. Zlatev, L. M. Wang and P. J. Steinhardt, Phys. Rev. Lett. 82, 896 (1999); P. J. Steinhardt, L. M. Wang and I. Zlatev, Phys. Rev. D 59, 123504 (1999); L. Amendola, Phys. Rev. D 62, $043511(2000)$.

[6] R. R. Caldwell, Phys. Lett. B 545, 23 (2002); S. Nojiri and S. D. Odintsov, Phys. Lett. B 562, $147(2003)$.

[7] V. Sahni and A. A. Starobinsky, Int. J. Mod. Phys. D 9, 373 (2000); P. J E. Peebles and B. Ratra, Rev. Mod. Phys. 75, 559 (2003); T. Padmanabhan, Phys. Rep. 380, 235 (2003); V. Sahni, Lect. Notes Phys. 653, 141 (2004); arXiv:astro-ph/0502032; E. J. Copeland, M. Sami and S. Tsujikawa, Int. J. Mod. Phys. D 15, 1753 (2006).

[8] U. Alam, V. Sahni, T. D. Saini and A. A. Starobinsky, Mon. Not. Roy. Ast. Soc. 354275 (2004); U. Alam, V. Sahni and A. A. Starobinsky, JCAP 0406008 (2004). 
[9] B. Feng, X. L. Wang and X. M. Zhang, Phys. Lett. B 607, 35 (2005) .

[10] B. Feng, M. Li, Y. S. Piao and X. Zhang, Phys. Lett. B 634, 101 (2006) ; Z. K. Guo, Y. S. Piao, X. M. Zhang and Y. Z. Zhang, Phys. Lett. B 608, 177 (2005); X. F. Zhang, H. Li, Y. S. Piao and X. M. Zhang, Mod. Phys. Lett. A 21, 231 (2006) ; M. Z. Li, B. Feng and X. M. Zhang, JCAP 0512, 002 (2005); Y.F. Cai, H. Li, Y.S. Piao, X.M. Zhang, Phys.Lett.B646, 141, (2007); Y.F. Cai, J. Wang, Class.Quant.Grav.25, 165014, (2008).

[11] H. Wei, R.G. Cai, D.F. Zeng, Class.Quant.Grav. 22, 3189 (2005) ; H. Wei, R.G. Cai, Phys.Lett. B634, 9 (2006) .

[12] X. Zhang, Commun.Theor.Phys.44, 762 (2005); Phys.Rev.D74, 103505, (2006) .

[13] I. Y. Arefeva, A. S. Koshelev and S. Y. Vernov, Phys. Rev. D 72, 064017 (2005); S. Y. Vernov, arXiv:astro-ph/0612487; A. S. Koshelev, JHEP 0704, 029 (2007).

[14] H. Mohseni Sadjadi, M. Alimohammadi, Phys. Rev. D 74, 043506 (2006); J. Sadeghi, M. R. Setare, A. Banijamali, F. Milani, Phys. Lett. B 662, 92 (2008); M. R. Setare, J. Sadeghi, A. R. Amani, Phys. Lett. B 660, 299 (2008); M. R. Setare, E. N. Saridakis, arXiv:0810.4775; M. R. Setare, E. N. Saridakis, arXiv:0809.0114.

[15] L.P. Chimento, M. Forte, R. Lazkoz, M.G. Richarte, arXiv:0811.3643; G. Leon, R. Cardenas, J.L. Morales, arXiv:0812.0830.

[16] Y.F. Cai, M.Z. Li, J.X. Lu, Y.S. Piao, T.T. Qiu, X.M. Zhang, Phys. Lett. B651, 1(2007).

[17] A Sen, JHEP 0204,048, (2002); JHEP 0207,065 (2002).

[18] G. W. Gibbons, Phys. Lett. B 537, 1 (2002); G. Shiu and I. Wasserman, Phys. Lett. B 541, 6 (2002); T. Padmanabhan and T. R. Choudhury, Phys. Rev. D 66, 081301 (2002); A.Frolov, L.Kofman, A. Starobinsky Phys.Lett.B545,8, (2002).

[19] M. Fairbairn and M. H. G. Tytgat, Phys. Lett. B 546, 1 (2002); L. Kofman and A. Linde, JHEP 0207, 004 (2002); M. Sami, Mod. Phys. Lett. A 18, 691 (2003); Y.S. Piao, R.G. Cai, X.M. Zhang, Y.Z. Zhang, Phys.Rev. D66 (2002) 121301 ; J. M. Cline, H. Firouzjahi and P. Martineau, JHEP 0211, 041 (2002); M. C. Bento, O. Bertolami and A. A. Sen, Phys. Rev. D 67, 063511 (2003); Y.S. Piao, Q.G. Huang, X.M. Zhang, Y.Z. Zhang, Phys.Lett. B570,1 (2003) ; Z.K. Guo, Y.S. Piao, R.G. Cai, Y.Z. Zhang, Phys.Rev. D68, 043508 (2003) ; S. Nojiri and S. D. Odintsov, Phys. Lett. B 571, 1 (2003);D. A. Steer and F. Vernizzi, Phys. Rev. D 70, 043527 (2004); V. Gorini, A. Y. Kamenshchik, U. Moschella and V. Pasquier, Phys. Rev. D 69, 123512 (2004); B. C. Paul and M. Sami, Phys. Rev. D 70, 027301 (2004); J. M. Aguirregabiria 
and R. Lazkoz, Mod. Phys. Lett. A 19, 927 (2004).

[20] T. Padmanabhan, Phys. Rev. D 66, 021301 (2002);A. Feinstein, Phys. Rev. D 66, 063511 (2002); J.S. Bagla, H. K. Jassal, and T. Padmanabhan, Phys. Rev. D 67, 063504 (2003);Gianluca Calcagni and Andrew R. Liddle, Phys.Rev.D74, 043528 (2006).

[21] D.Choudhury, D. Ghoshal, D. P. Jatkar, and S. Panda, Phys. Lett. B 544, 231 (2002); X.Z Li, J.G. Hao, D.J. Liu, Chin.Phys.Lett. 19 1584, (2002); J.G. Hao and X.Z. Li, Phys. Rev. D 66, 087301 (2002); M. R. Garousi, M. Sami, and S. Tsujikawa, Phys. Rev. D 70, 043536 (2004); V. H. Cardenas, Phys. Rev. D 73, 103512 (2006).

[22] J. M. Aguirregabiria and R. Lazkoz, Phys. Rev. D 69, 123502 (2004).

[23] E. J. Copeland, M. R. Garousi, M. Sami, and S. Tsujikawa, Phys. Rev. D 71, 043003 (2005).

[24] J.G. Hao, X.Z. Li, Phys. Rev. D 68, 043501 (2003).

[25] S. Tsujikawa, M. Sami Phys. Lett. B 603, 113 (2004).

[26] E.J. Copeland, A.R. Liddle, and D. Wands, Phys. Rev. D 57, 4686 (1998).

[27] Imogen P. C. Heard and David Wands, Class. Quant. Grav. 19, 5435 (2002).

[28] R. Bean, S.H. Hansen, and A. Melchiorri, Phys. Rev. D 64, 103508 (2001).

[29] Z.K. Guo, Y.S. Piao, and Y.Z. Zhang, Phys. Lett. B 568, 1 (2003).

[30] H. Kodama and M. Sasaki, Prog. Theor. Phys. Suppl. 78, 1 (1984).

[31] J.Q.Xia, Y.F.Cai, T.T.Qiu, G.B.Zhao, and X.M.Zhang, Int.J.Mod.Phys. D17,1229 (2008).

[32] J.K. Erickson, R.R. Caldwell, P.J. Steinhardt, C. Armendariz-Picon, and V. Mukhanov, Phys. Rev. Lett. 88,121301(2002);C. Armendariz-Picon, E. A. Lim, JCAP 0508:007, (2005); J.-P. Bruneton, Phys. Rev. D75:085013, (2007); Jin U Kang, Vitaly Vanchurin, Sergei Winitzki Phys.Rev.D76:083511,(2007); E. Babichev, V. Mukhanov, A. Vikman, JHEP 0802:101,(2008). 